\title{
Gyrokinetic modelling of stationary electron and impurity profiles in tokamaks
}

\author{
A. Skyman, ${ }^{\text {a) }}$ D. Tegnered, ${ }^{\text {b) }}$ H. Nordman, ${ }^{\text {c) }}$ and P. Strand ${ }^{\text {d) }}$ \\ Euratom-VR Association, Department of Earth and Space Sciences, Chalmers University of Technology, \\ SE-412 96 Göteborg, Sweden
}

(Received 21 February 2014; accepted 21 July 2014; published online 11 September 2014)

Particle transport due to Ion Temperature Gradient (ITG)/Trapped Electron Mode (TEM) turbulence is investigated using the gyrokinetic code GENE. Both a reduced quasilinear treatment and nonlinear simulations are performed for typical tokamak parameters corresponding to ITG dominated turbulence. The gyrokinetic results are compared and contrasted with results from a computationally efficient fluid model. A selfconsistent treatment is used, where the stationary local profiles are calculated corresponding to zero particle flux simultaneously for electrons and trace impurities. The scaling of the stationary profiles with magnetic shear, safety factor, electron-to-ion temperature ratio, collisionality, toroidal sheared rotation, plasma $\beta$, triangularity, and elongation is investigated. In addition, the effect of different main ion mass on the zero flux condition is discussed. The electron density gradient can significantly affect the stationary impurity profile scaling. It is therefore expected that a selfconsistent treatment will yield results more comparable to experimental results for parameter scans where the stationary background density profile is sensitive. This is shown to be the case in scans over magnetic shear, collisionality, elongation, and temperature ratio, for which the simultaneous zero flux electron and impurity profiles are calculated. A slight asymmetry between hydrogen, deuterium, and tritium with respect to profile peaking is obtained, in particular, for scans in collisionality and temperature ratio.

[http://dx.doi.org/10.1063/1.4894739]

\section{INTRODUCTION}

It is well known that the shape of the main ion density and impurity profiles is crucial for the performance of a fusion device. Inward peaking of the main ion (electron) density profile is beneficial for the fusion performance since it enhances the fusion power production. For impurities on the other hand, a flat or hollow profile is preferred, since impurity accumulation in the core leads to fuel dilution and radiation losses which degrades performance.

The particle profiles are determined by a balance between particle sources and particle fluxes, a subject that historically has been given much less attention than energy transport and the associated temperature profiles. Hence, electron density profiles are often treated as a parameter in theoretical studies of transport rather than being selfconsistently calculated.

Turbulent transport in the core of tokamaks is expected to be driven mainly by Ion Temperature Gradient (ITG) and Trapped Electron (TE) modes. Particle transport driven by ITG/TE mode turbulence has been investigated in a number of theoretical studies. ${ }^{1-26}$ Most work in this area has been focused on either calculations of stationary electron profiles or on impurity transport using prescribed electron density profiles.

It is well established theoretically that turbulent particle transport in tokamaks has contributions from both diagonal (diffusive) and non-diagonal (convective) terms. The nondiagonal transport contributions may give rise to an inward

\footnotetext{
a)andreas.skyman@chalmers.se

b) tegnered@chalmers.se

c)hans.nordman@chalmers.se

d) par.strand@chalmers.se. URL: http://www.chalmers.se/rss/.
}

pinch which can support an inwardly peaked profile even in the absence of particle sources in the core. The stationary peaked profile is then obtained from a balance between diffusion and convection.

It is known that the electron density gradient can significantly affect the stationary impurity profile scaling. ${ }^{24}$ In the present work, therefore, the background electron density and impurity peaking is treated selfconsistently, by simultaneously calculating the local profiles corresponding to zero turbulent particle flux of both electrons and impurities.

Linear and nonlinear gyrokinetic simulations using the code GENE $^{27}$ are employed. ${ }^{28,29}$ The scaling of the stationary profiles with key plasma parameters like magnetic shear, temperature ratio and temperature gradient, toroidal sheared rotation, safety factor, and collisionality is investigated for a deuterium (D) plasma. The isotope scaling of stationary profiles for hydrogen $(\mathrm{H})$, tritium $(\mathrm{T})$, and 50/50 DT plasmas is also studied. The gyrokinetic results are compared and contrasted with results from a computationally efficient fluid model,${ }^{30}$ suitable for use in transport codes such as JETTO, where it is currently implemented. ${ }^{31}$

The parameters are varied around Cyclone Base Case $\left(\mathrm{CBC}^{32}\right)$ parameters, thereby covering a wide range of tokamak scenarios. The CBC is an ITG mode dominated $\mathrm{H}$-mode discharge and, though set far from marginal stability, is an interesting case for study, and is widely used as a testing ground and benchmark for theoretical and numerical studies. The CBC parameters, with $\mathrm{D}$ as main ions in the present study, are listed in Table I.

The rest of the paper is organised as follows: In Sec. II, the gyrokinetic and fluid models used in this paper are 
introduced; in Sec. III, a theoretical background is given, including considerations regarding analysis and numerics. The main results are presented in Sec. IV, where scalings of the stationary profiles for electrons and impurities are discussed; results for background peaking of different main ion isotopes are presented and discussed in Sec. V; finally, in Sec. VI, follow the concluding remarks.

\section{MODELS}

\section{A. GENE}

The gyrokinetic simulations in this study were performed using the gyrokinetic turbulence code GENE. ${ }^{28,29}$ This is an Eulerian-type code of the $\delta f$ variety (i.e., employing a fixed grid in phase space, and considering the perturbed part of the gyrokinetic distribution function), which allows for an arbitrary number of species. Both quasi- and nonlinear simulations including kinetic ions, electrons, and impurities were performed. All impurities were treated as fully kinetic species with low concentrations. For studying the effects of collisions, we considered the GENE implementation of the Landau-Boltzmann type collision operator. ${ }^{29}$

For the simulation domain, a flux tube with periodic boundary conditions in the perpendicular plane was used. The nonlinear (NL) simulations were performed using a $96 \times 96 \times 32$ grid in the normal, bi-normal, and parallel spatial directions, respectively; in the parallel and perpendicular momentum directions, a $48 \times 12$ grid was used. For the linear and quasilinear computations, a typical resolution was $12 \times 24$ grid points in the parallel and normal directions, with $64 \times 12$ grid points in momentum space. The nonlinear simulations were typically run up to $t=300 R / c_{\mathrm{s}}$, where $R$ is the major radius and $c_{\mathrm{s}}=\sqrt{T_{e} / m_{i}}$.

\section{B. Fluid model}

A brief summary of the fluid model is given here, for a detailed description, see Refs. 22 and 30 and references therein. Each species (main ions, electrons, and impurities) is described by the continuity, parallel momentum, and energy equations for the perturbations in density, parallel velocity, and temperature. The parallel velocity perturbation is zero for the bounce averaged trapped electron fluid, and

TABLE I. Parameters for the CBC. ${ }^{32}$

\begin{tabular}{lc}
\hline \hline$r / R$ & 0.18 \\
$\hat{s}$ & 0.796 \\
$q_{0}$ & 1.4 \\
$R / L_{n_{i, e}}$ & 2.22 \\
$R / L_{T_{i, e}}$ & 6.96 \\
$T_{i} / T_{e}$ & 1.0 \\
$T_{e}(\mathrm{keV})$ & 2.85 \\
$n_{e}\left(10^{19} \mathrm{~m}^{-3}\right)$ & 3.51 \\
$B_{0}(\mathrm{~T})$ & 3.1 \\
$R(\mathrm{~m})$ & 1.65 \\
$\beta(\%)^{\mathrm{a}}$ & 0.42 \\
$\nu_{e i}\left(c_{s} / R\right)^{\mathrm{a}}$ & 0.05 \\
\hline \hline
\end{tabular}

${ }^{\mathrm{a}}$ Denotes derived parameters. effects of finite-Larmor-radius (FLR) have been neglected for electrons and impurities.

The ion, impurity, and electron perturbations are coupled through the quasineutrality condition $\delta n_{i} / n_{i}+Z f_{Z} \delta n_{Z} / n_{Z}$ $=f_{t} \delta n_{e t} / n_{e t}+\left(1-f_{t}\right) \delta n_{e t} / n_{e t}$, where $f_{t}=\sqrt{2 \varepsilon /(1+\varepsilon)}$ is the fraction of trapped electrons, and $f_{Z}$ the fraction of impurities, $n_{i}, n_{Z}, n_{e t}$, and $n_{e f}$ are the densities of the main ions, impurities, trapped, and free electrons, respectively. The free electrons are assumed to be Boltzmann distributed. The resulting eigenvalue equation is in the present study reduced to a set of coupled algebraic equations by assuming a strongly ballooning eigenfunction $(\phi=(1+\cos \theta) / \sqrt{3},|\theta|<\pi){ }^{33}$ The operators $k_{\|}, k_{\perp}$, and the magnetic drift $\omega_{D j}$ are then replaced by averages over the strongly ballooning eigenfunction as

$$
\begin{gathered}
\left\langle k_{\|}^{2}\right\rangle=\frac{1}{3}(q R)^{2}, \\
\left\langle k_{\perp}^{2}\right\rangle=k_{\theta}^{2}\left(1+\left(\frac{\pi^{2}}{3}-\frac{5}{2}\right) \hat{s}^{2}\right), \\
\left\langle\omega_{D i, Z}\right\rangle=\omega_{D_{0} i, Z}\left(\frac{2}{3}+\frac{5}{9} \hat{s}\right),
\end{gathered}
$$

where $\hat{s}$ is the magnetic shear, $q$ is the safety factor, $R$ is the major radius, and the average over the eigenfunction is defined as $\langle\ldots\rangle=\int_{-\pi}^{\pi} \phi(\ldots) \phi \mathrm{d} \theta$. This analysis is expected to give results in qualitative agreement with the full eigenvalue solution if magnetic shear is not too small.

The particle and heat fluxes are calculated assuming linear relations between the field quantities combined with a modified mixing length estimate of the turbulence saturation level. ${ }^{30}$ The fluxes are computed numerically by adding the separate transport contributions from the ITG and TE modes at a fixed wavenumber, assuming isotropic turbulence with $k_{\theta} \rho_{\mathrm{s}} \approx k_{r} \rho_{\mathrm{s}} \approx 0.2-0.3$.

\section{STATIONARY PROFILES}

The local particle transport for species $j$ can be formally divided into its diagonal and off-diagonal parts

$$
\frac{R \Gamma_{j}}{n_{j}}=D_{j} \frac{R}{L_{n_{j}}}+D_{T_{j}} \frac{R}{L_{T_{j}}}+R V_{p, j} .
$$

Here, the first term on the right hand side is the diffusion and the second and third constitute the off-diagonal pinch. The first of the pinch terms is the particle transport due to the temperature gradient (thermo-diffusion) and the second is the convective velocity, which includes contributions from curvature, parallel compression and roto-diffusion. In Eq. (4), $R / L_{X_{j}} \equiv-R \nabla X_{j} / X_{j}$ are the local gradient scale lengths of density and temperature, normalised to the major radius $(R)$. In general, the transport coefficients dependent on the gradients, though in the trace impurity limit the transport is linear in both $R / L_{n_{Z}}$ and $R / L_{T_{Z}}$. A review of the offdiagonal contributions is given in Ref. 34 .

At steady state, the contributions from the different terms in the particle transport will tend to cancel if the particle sources are small, resulting in zero particle flux. Solving 
Eq. (4) for zero particle flux, with $V_{j}=D_{T_{j}} 1 / L_{T_{j}}+V_{p . j}$ yields

$$
\left.P F_{j} \equiv \frac{R}{L_{n_{j}}}\right|_{\Gamma=0}=-\frac{R V_{j}}{D_{j}},
$$

which is the steady state gradient of zero particle flux for species $j$. This measure quantifies the balance between diffusion and advection, and gives a measure of how "peaked" the local density profile is at steady state. It is therefore referred to as the "peaking factor" and denoted $P F_{j}$.

In order to investigate the transport, NL GENE simulations were performed from which $P F_{e}$ for the stationary electron profiles were calculated. The results were compared with quasilinear (QL) results, also obtained using GENE. The background peaking factor was found by explicitly seeking the gradient of zero particle flux by calculating the electron flux for several values of the density gradient. A typical set of simulations is displayed in Fig. 1(a), where the time evolution of the electron flux for three density gradients near the gradient of zero particle flux is shown (fluxes are in gyro-Bohm units, with $D_{\mathrm{GB}}=c_{\mathrm{s}} \rho_{\mathrm{s}}^{2} / R$ ). A second order polynomial $p$ was then fitted to the data closest to the zero flux gradient and then the $P F_{e}$ was found as the appropriate root of $p$. The error for $P F_{e}$ was approximated by finding the corresponding roots of $p \pm \max \left[\sigma_{\Gamma}\right]$, and using the difference between these roots as a measure of the error. In Fig. 1(b), the particle flux spectrum for a NL GENE simulation for CBC near this gradient is shown, together with the corresponding spectrum from the fluid model. The figure illustrates that the total flux is zero due to a balance of inward and outward transports occurring at different wavenumbers. This represents a challenge for reduced models using a fixed wave number, such as the fluid model and QL GENE as used here. The method for finding $P F_{e}$ from the QL GENE simulations is the same as that described above, but here a reduced treatment was used, that includes only the dominant mode, which is an ITG mode for CBC-like parameters.

In the trace impurity limit, i.e., when the fraction of impurities is sufficiently small, the impurity dynamics do not affect the turbulence dynamics. Therefore, when finding the simultaneous peaking factor of the background and impurities, the former can be found first and used in the simulations of the latter without loss of generality. Furthermore, in the trace impurity limit, the transport coefficients of Eq. (4) for trace impurities do not depend on the species' gradients of density and temperature, meaning that (4) is a linear relation in those gradients. This means that the impurity peaking, as well as the contribution to $P F_{Z}$ from the thermodiffusion $\left(P F_{T}\right)$ and the convective velocity $\left(P F_{p}\right)$, can be found from simulations with appropriately chosen gradients using the method outlined in Ref. 35. The peaking factors are calculated for several impurity species, using the reduced QL GENE model. The impurity peaking factors were also studied using the fluid model, but were seen not to differ significantly from gyrokinetic results and have been left out of the following figures for clarity. The difference in impurity peaking factors between NL GENE, QL GENE, and the present fluid model has been covered in previous works. ${ }^{22-24,26}$

The simulations have been performed in a circular equilibrium with aspect ratio $R / a=3$, using kinetic ions, electrons, and impurities, except when studying the effects of shaping. Then, the Miller equilibrium model was used instead. ${ }^{36}$ Impurities were included at trace amounts $\left(n_{Z} / n_{e}=10^{-6}\right.$ ), so as not to affect the turbulent dynamics. The impurity mass was assumed to be $A_{Z}=2 Z$, where $Z$ is the charge number. The dynamics were further assumed to be electrostatic $(\beta \approx 0)$, except when studying the effect of $\beta$ on the stationary profiles.

\section{SIMULTANEOUS STATIONARY PROFILES OF ELECTRONS AND IMPURITIES}

First, we examine the dependence of the heat transport and of $P F_{e}$ on the ion temperature gradient. The result is shown in Fig. 2, where the ion energy transport from NL GENE simulations is displayed, together with electron peaking factors from NL GENE, QL GENE, and fluid simulations. The QL GENE and fluid results are obtained for two different wavenumbers, corresponding to the typical scales of maximum growth rate $\left(k_{\theta} \rho_{s}=0.3\right)$ and fluctuation amplitude $\left(k_{\theta} \rho_{s}=0.2\right)$, respectively. In the TE mode dominated region $\left(R / L_{T_{i}} \lesssim 4.5\right)$, the peakedness of the background profile shows a steady increase with increasing ion temperature gradient, while the ion heat transport is negligible until the ITG mode is destabilised. Simulations with impurities using the obtained stationary background gradient were then performed, choosing $R / L_{T_{Z}}=R / L_{T_{i}}$. The impurities (Be $(Z=4), \mathrm{C}(Z=6), \mathrm{Ne}(Z=10)$, and $\mathrm{Ni}(Z=28))$ show the same behaviour, but are considerably less peaked than the background, at most by a factor of 0.5 , as illustrated in Fig.

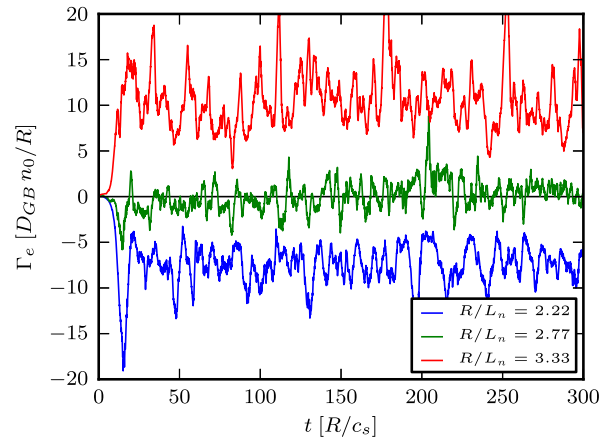

(a): electron particle flux for different density gradients

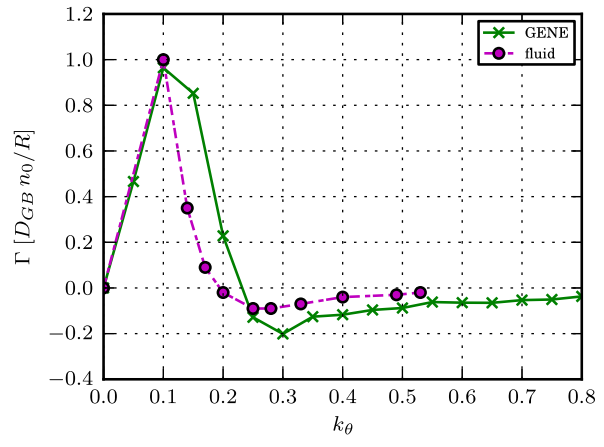

(b): poloidal wavenumber spectra of the electron particle flux, evaluated at the gradient of zero flux
FIG. 1. Time series and spectra of electron particle transport for $\mathrm{CBC}$ with $\mathrm{D}$ as main ions near to the zero flux gradient $\left(R / L_{n_{e}}=2.77\right)$; obtained from NL GENE simulations. A flux spectrum from the fluid model, renormalised to a peak value of 1 , is displayed with the NL GENE spectrum, and shows the same features. 


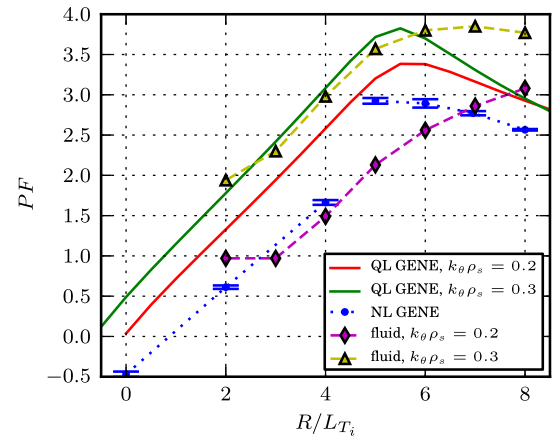

(a): background peaking factors $\left(P F_{e}\right)$

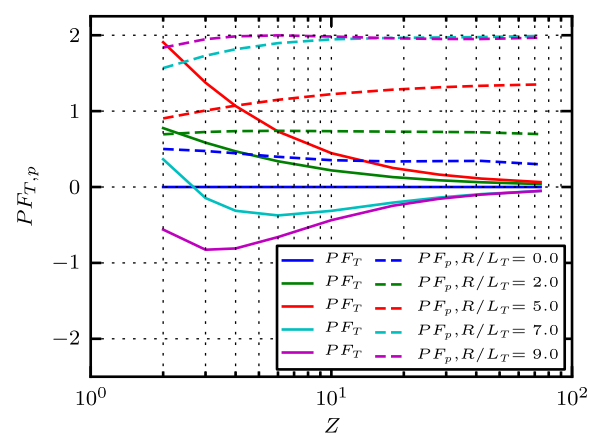

(c): contributions to $P F_{Z}$ from thermopinch $\left(P F_{T}\right)$

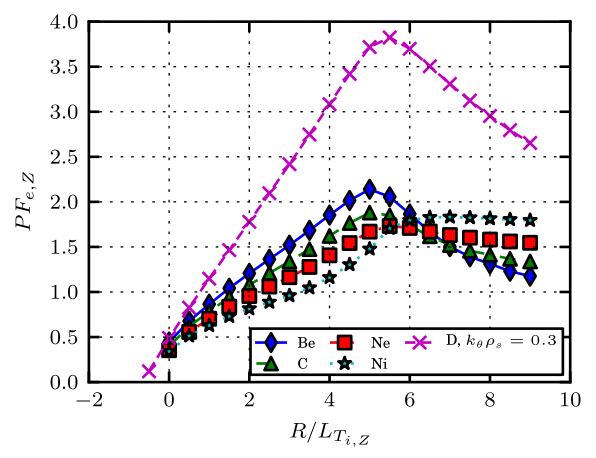

(b): simultaneous QL GENE scalings of $P F_{e}$ and

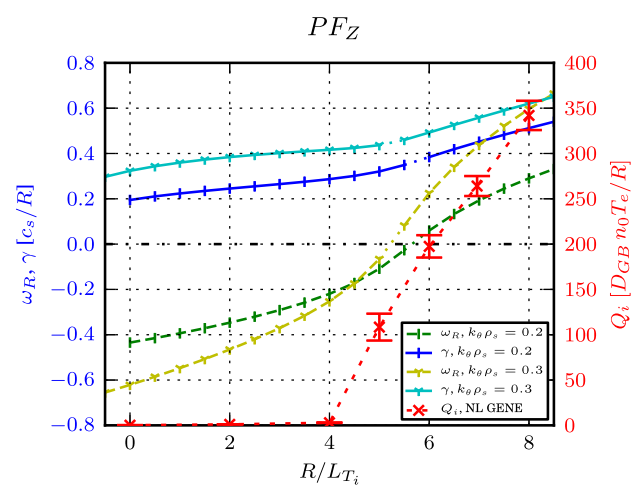

(d): heat flux and eigenvalues
FIG. 2. Scaling of background electron peaking, impurity peaking, ion heat flux, and eigenvalues with $R / L_{T_{i}}$.

and pure convection $\left(P F_{p}\right)$ vs. impurity charge

2(b). Fig. 2(c) illustrates the difference between the TE and ITG mode scalings: in the TE mode region, both parts of $P F_{Z}$ are inward, but while the pure convection grows steadily stronger with increasing $R / L_{T_{i, Z}}$, the thermopinch contribution decreases in magnitude and eventually changes direction from inward to outward once $R / L_{T_{i, Z}}$ is sufficiently above the ITG threshold. Above the threshold, the impurities with higher $Z$ show less sensitivity to changing $R / L_{T_{i, Z}}$, which can be attributed to the dominance for these impurities of the pure convection part of the peaking factor, which quickly saturates at $P F_{p, Z} \approx 2$ above the ITG threshold for all impurities. For the impurities with lower charge numbers, on the other hand, the peaking factor is dominated by the thermopinch $(\sim 1 / Z)$, which shows an increasing outward tendency as the temperature gradient steepens. Though the ion energy transport shows a stiff increase with the driving gradient above the ITG threshold, only a moderate reduction is seen in the peaking factor. The TE mode was seen to pass smoothly into to the ITG mode, when following zero-flux density gradient, as shown in Fig. 2(d). The temperature gradient where the mode changes direction therefore corresponds to the real frequency $\omega_{R}=0$. For both ITG and TE modes, $\left|\omega_{R}\right| \rightarrow 0$ leads to the highest background peaking as shown in Fig. 2(a) and discussed in Ref. 17. The fluid model was seen to give the best quantitative agreement with the nonlinear GENE results for $k_{\theta} \rho_{s}=0.2$, while the tendency was better reproduced for $k_{\theta} \rho_{s}=0.3$.

It is worth noting that the steady state peaking found in the simulations is considerably higher than that in the original CBC experiment $\left(R / L_{n_{e, i}}=2.22\right)$. As is known, ${ }^{3,14,17,37}$ this is due to the neglect of collisions, as they normally are in the $\mathrm{CBC}$. The collisionality for the $\mathrm{CBC}$ parameters is $\nu_{e i} \approx 0.05 c_{\mathrm{s}} / R$, which is of the same order as the growthrates and real frequencies observed, and collisions can be expected to have a notable impact on the transport. When collisions are added, the background peaking factor is indeed lowered to a level consistent with the prescribed background gradient for the CBC, as seen in Fig. 5(a).

The electron peaking factor is reduced with increasing ion-electron temperature ratio $\left(T_{i} / T_{e}\right)$ for $\mathrm{CBC}$ parameters, as can be seen in Fig. 3. As with the temperature gradient, the NL GENE results show a strong increase in the ion heat transport, but only a weak scaling of $P F_{e}$, while the trend is more pronounced for the QL GENE simulations. The fluid model shows a good quantitative agreement with the NL GENE results for $k_{\theta} \rho_{s}=0.2$, while better reproducing the trend for $k_{\theta} \rho_{s}=0.3$. The discrepancy between the NL and QL GENE results may be a result of the QL GENE treatment, which only includes the dominant mode, while the contribution from the subdominant TE mode is non-negligible for low values of $T_{i} / T_{e}$, resulting in a more moderate scaling for the NL GENE and fluid models. In Fig. 3(b), the selfconsistently obtained quasilinear peaking of electrons and impurities is shown. Impurities with lower charge numbers $(Z)$, as well as the background, show the same dependence on $T_{i} / T_{e}$, with a decrease in the peaking as the ion temperature is increased, and a weaker tendency for smaller wavenumbers. For the impurities with higher $Z$, on the other hand, increased ion temperature leads to slightly more peaked impurity profiles. In Fig. 3(c), it is shown that the effect for the impurities is mainly due to an increase in the relative contribution from the outward thermopinch $(\sim 1 / Z)$ with increased ion temperature, which affects 


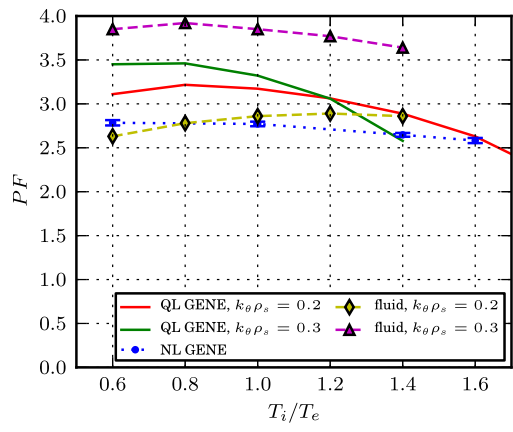

(a): background peaking factors $\left(P F_{e}\right)$

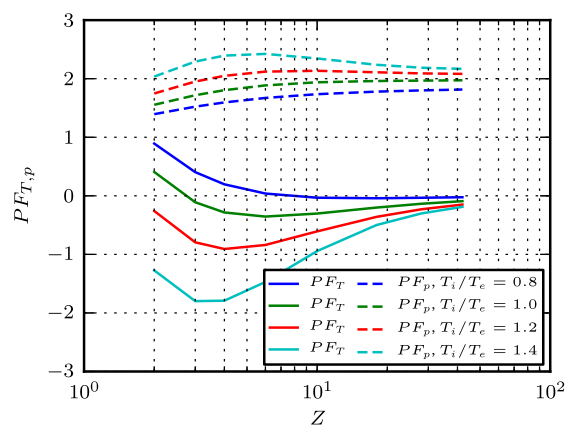

(c): contributions to $P F_{Z}$ from thermopinch $\left(P F_{T}\right)$

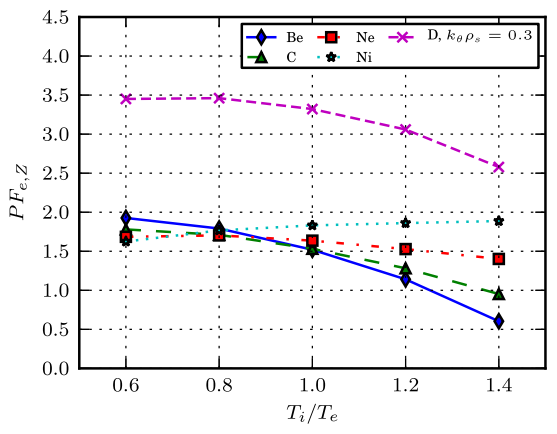

(b): simultaneous QL GENE scalings of $P F_{e}$ and

$P F_{Z}$

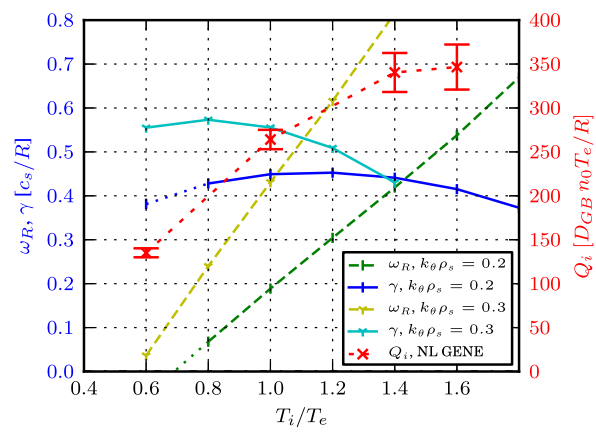

(d): heat flux and eigenvalues
FIG. 3. Scaling of background electron peaking, impurity peaking, ion heat flux, and linear eigenvalues with $T_{i} / T_{e}$.

and pure convection $\left(P F_{p}\right)$ vs. impurity charge

the low $Z$ impurities more strongly. To first order, the thermopinch is proportional to the real frequency. As seen in Fig. 3(d) it increases with increasing $T_{i} / T_{e}$, which explains its increasing importance for higher ion temperatures. As was the case for the scaling with $R / L_{T_{i}}, P F_{e}$ is maximised for $\omega_{R} \approx 0$, as seen when comparing Figs. 3(a) and 3(d).

In Fig. 4(a), the scaling with magnetic shear $(\hat{s})$ is studied. The electron peaking shows a strong and near linear

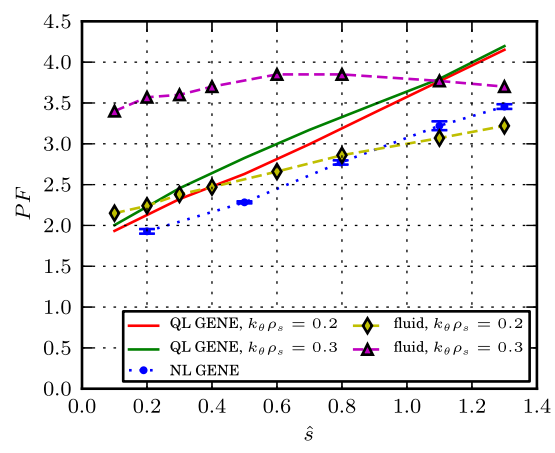

(a): background peaking factors $\left(P F_{e}\right)$

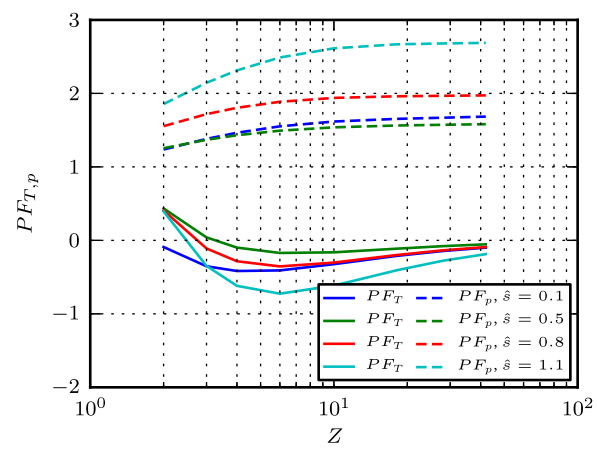

(c): contributions to $P F_{Z}$ from thermopinch $\left(P F_{T}\right)$ and pure convection $\left(P F_{p}\right)$ vs. impurity charge

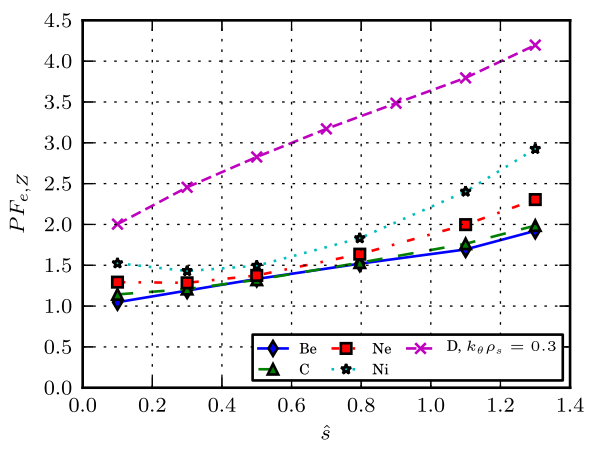

(b): simultaneous QL GENE scalings of $P F_{e}$ and $P F_{Z}$

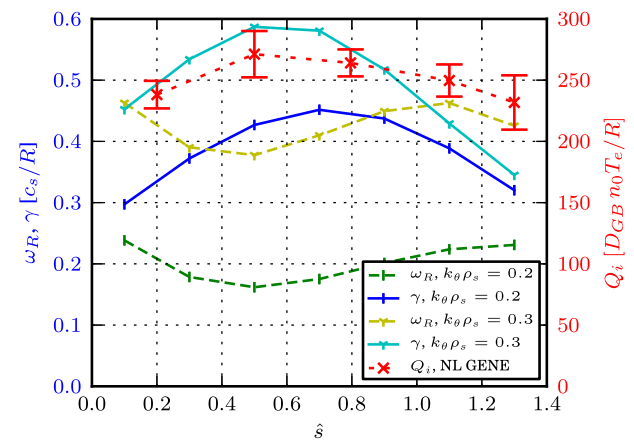

(d): heat flux and eigenvalues
FIG. 4. Scaling of background electron peaking, impurity peaking, ion heat flux, and linear eigenvalues with $\hat{s}$. 


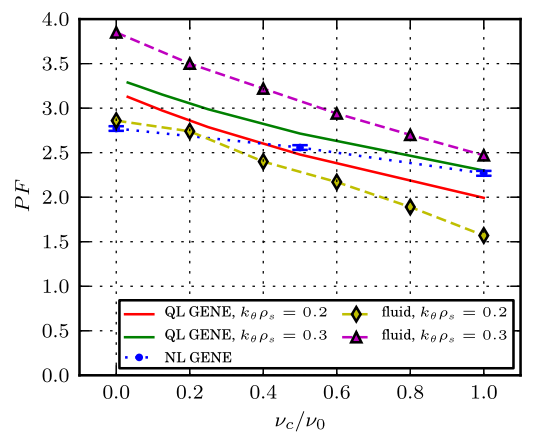

(a): background peaking factors $\left(P F_{e}\right)$

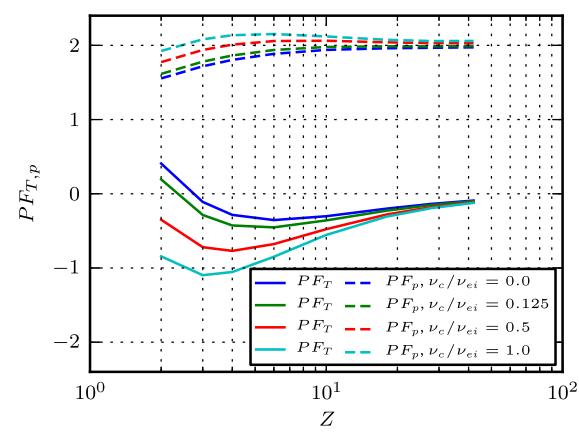

(c): contributions to $P F_{Z}$ from thermopinch $\left(P F_{T}\right)$

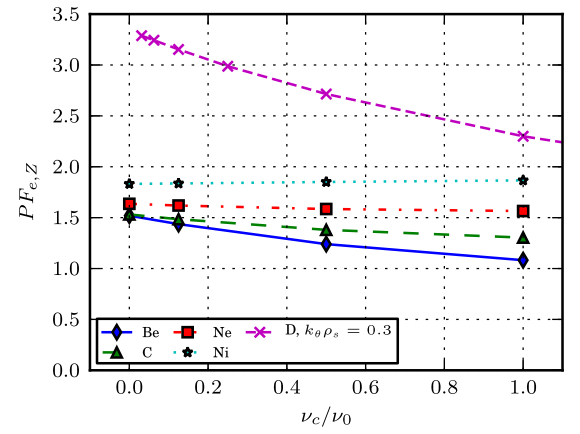

(b): simultaneous QL GENE scaling of $P F_{e}$ and $P F_{Z}$

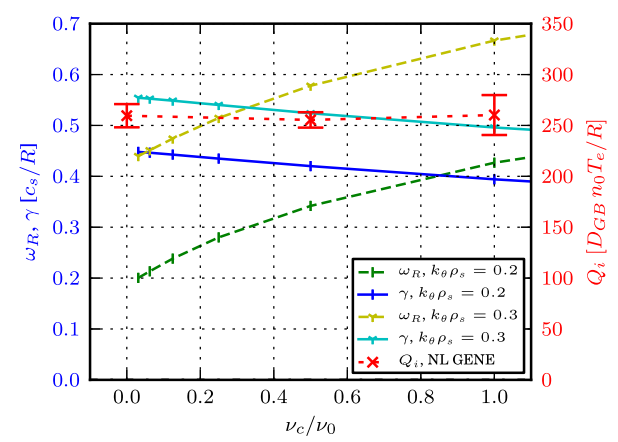

(d): heat flux and eigenvalues
FIG. 5. Scaling of background electron peaking, impurity peaking, ion heat flux, and linear eigenvalues with $\nu_{e i}$.

and pure convection $\left(P F_{p}\right)$ vs. impurity charge

dependence on $\hat{s}$, while the heat transport is only decreased slightly with increasing magnetic shear. This is similar to the results reported in Ref. 17 and is due to the shear dependence of the curvature pinch. This trend is as strong in both the QL and NL GENE simulations; however, the fluid results show quantitative and qualitative agreement mostly for $k_{\theta} \rho_{s}=0.2$. The effect of shear on the linear eigenvalues is not monotonic, with a destabilisation in the low to medium shear region followed by stabilisation as $\hat{s}$ is increased further. The selfconsistent results are shown in Fig. 4(b). For the impurities, the change in peaking factors due to magnetic shear follows the trend seen for the electrons, and impurities with higher $Z$ are more strongly affected. This is seen in Fig. 4(c) to be due mainly to a stronger inward convective pinch with increasing shear.

Next we cover the effect of electron-ion collisions on the peaking factors. Collisionality is known to affect the background by reducing the peaking factor..$^{3,13,14,17}$ This is shown in Fig. 5(a), where a reduction in $P F_{e}$ with increasing collisionality for all three models is seen, but little or no effect on the ion heat flux (Fig. 5(d)). The fluid model here shows a stronger dependence on the collisionality than the gyrokinetic models, and best reproduces the NL GENE results at low collisionality using $k_{\theta} \rho_{s}=0.2$ for low collisionality, while $k_{\theta} \rho_{s}=0.3$ agrees better for high collisionality. In Fig. 5(b), the selfconsistent results for a range of collisionalities are shown. The reduction in peaking factors with collisionality is also seen for low- $Z$ impurities, while the high- $Z$ impurities show little or no change in peaking due to collisions. The effect on the impurities is mainly due to an increase in the outward thermopinch $(\sim 1 / Z)$ with increased collisionality (Fig. 5(c)), due to a change of the real frequency as illustrated in Fig. 5(d).
The influence of sheared toroidal flows on the selfconsistent impurity peaking was also studied. Only purely toroidal rotation was considered, included through the $\boldsymbol{E} \times \boldsymbol{B}$ shearing rate, defined as $\gamma_{E}=-\frac{r}{q} \frac{1}{R} \frac{\partial v_{\text {tor }}}{\partial r}$. Hence, we consider flow shear in the limit where the flow is small, neglecting effects of centrifugal and Coriolis forces. These may, however, be important for heavier impurities. ${ }^{15}$ The results are shown in Fig. 6(a), where it can be seen that impurities are much more strongly affected by the rotation than the background, due to the difference in thermal velocity between main ions and impurities. For large values of $\gamma_{E}$, a strong decrease in impurity peaking is seen. The effect is due to the outward rotopinch which becomes important for large values of $\gamma_{E}$, as shown in Fig. 6(b). As with the shearing rate, this effect is more pronounced for high- $Z$ impurities, since the thermopinch dominates for low $Z$ values. In ASDEX $U$ roto-diffusion has been found to be a critical ingredient to include in order to reproduce the Boron profiles seen in experiments. ${ }^{21,25}$

Next the impact of electro magnetic effects was studied. As shown in Fig. 6(c), an increase in $\beta$ leads to a considerably lower background peaking, with $P F_{e}$ decreasing to a level comparable to $P F_{Z}$ for the higher $Z$ impurities for $\beta$ of the order of the experimental value $(\beta \gtrsim 0.42 \%)$. The self consistent impurity profiles are less affected, with a decreased $P F_{Z}$ for low $Z$ impurities, due again to an increase in the inward thermopinch, as seen in Fig. 6(d). The peaking factors for the higher charged impurities were less sensitive to changes in $\beta$, though the impurities with the highest values of $Z$ showed a slight increase in $P F_{Z}$, owing to an increase in the pure convection for high values of $\beta$. The results are consistent with those discussed in Refs. 20 and 38.

Finally, shaping effects were studied using the Miller equilibrium model. The quasi-linear electron peaking factor 


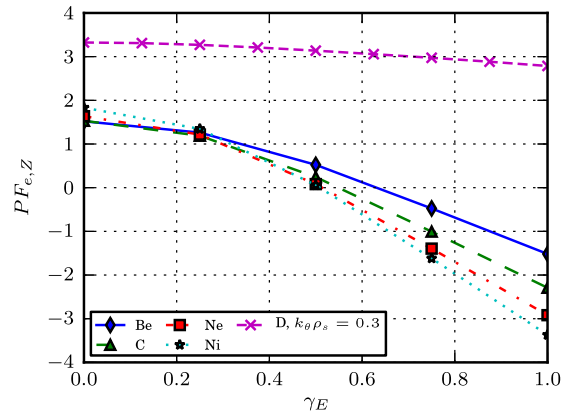

(a):

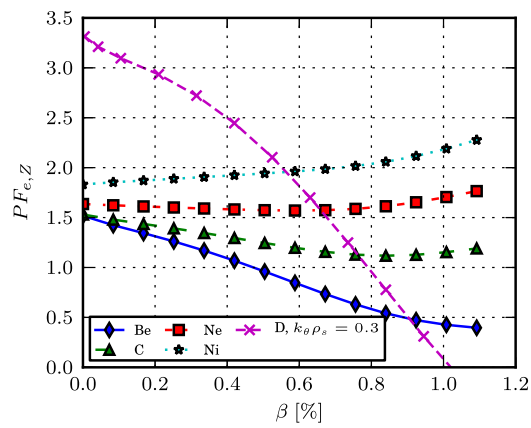

(c):

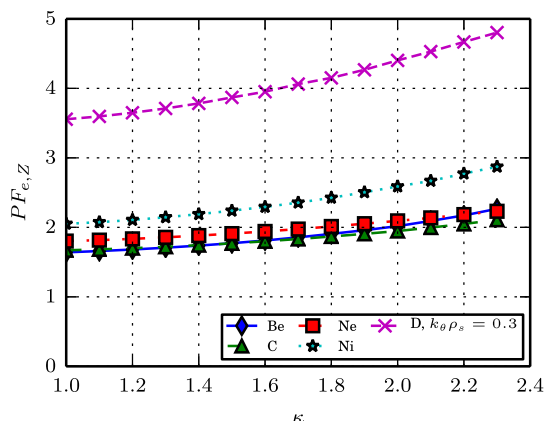

(e): simultaneous QL GENE scalings of $P F_{e}$ and $P F_{Z}$

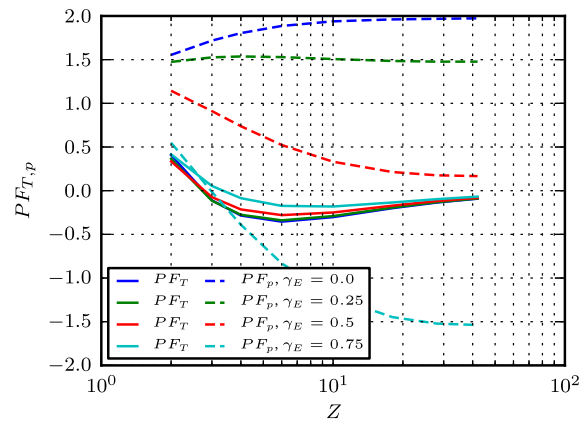

(b):

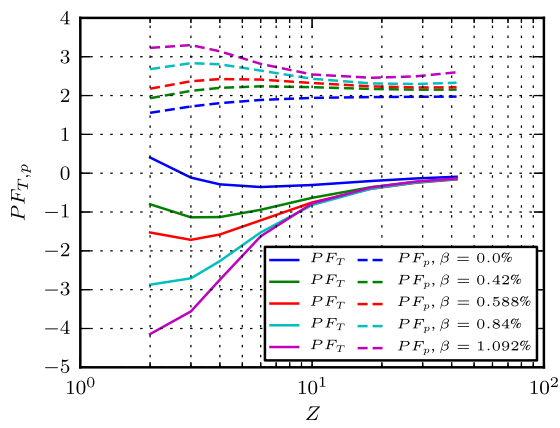

(d)

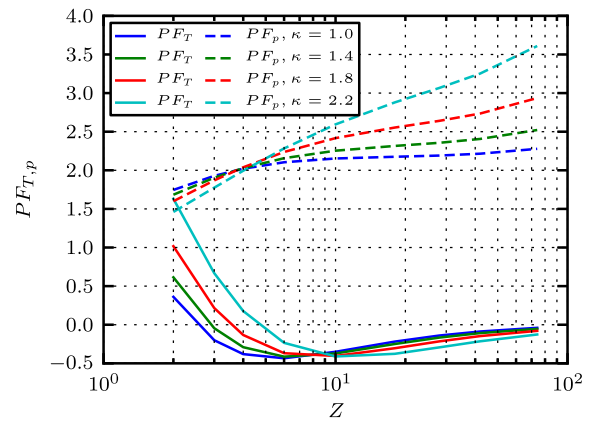

(f): contributions to $P F_{Z}$ from thermopinch $\left(P F_{T}\right)$ and pure convection $\left(P F_{p}\right)$ vs. impurity charge
FIG. 6. Scaling of background electron and impurity peaking with $\boldsymbol{E} \times \boldsymbol{B}$ shearing rate ((a) and (b)), elongation ((c) and (d)), and $\beta$ ((e) and (f)). as well as the self-consistent impurity peaking factors increase with higher elongation $(\kappa)$ as shown in Fig. 6(e). For impurities with low charge number, the increase in peaking is mainly due to a larger inward thermopinch while for high- $Z$ impurities it is caused by an increased pure convection, as seen in Fig. 6(f).

The dependence of the selfconsistent peaking factors on the safety factor $\left(q_{0}\right)$ and triangularity $(\delta)$ was also studied, and the scalings were found to be very weak.

\section{ISOTOPE EFFECTS ON THE BACKGROUND PEAKING}

The $\mathrm{CBC}$ prescribes hydrogen ions as the main ions, however, for future fusion power plants, a deuterium/tritium mixture will be used. Due to the difference in mass, it is known that $\mathrm{D}$ and $\mathrm{T}$ plasmas will behave differently from pure $\mathrm{H}$ plasmas. Differences in steady state peaking factors are expected, since both collisions and non-adiabatic electrons can break the gyro-Bohm scaling. ${ }^{39}$ To get an insight into the effect of the main ion isotope, the scalings for the

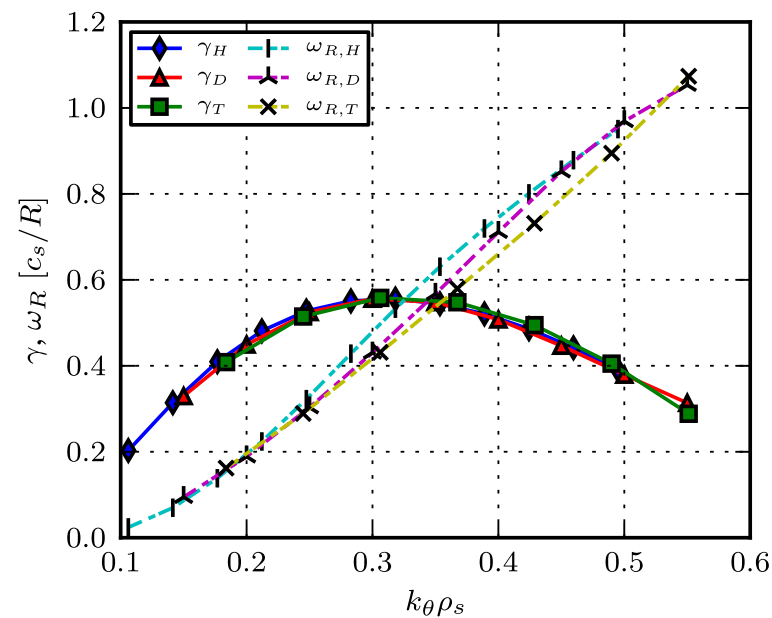

FIG. 7. Eigenvalue spectra (ITG) for CBC parameters (Table I), for H, D, and $\mathrm{T}$ as main ions, with $k_{\theta} \rho_{\mathrm{s}}$ and eigenvalues in species units $\left(c_{\mathrm{s}} / R\right)$. 


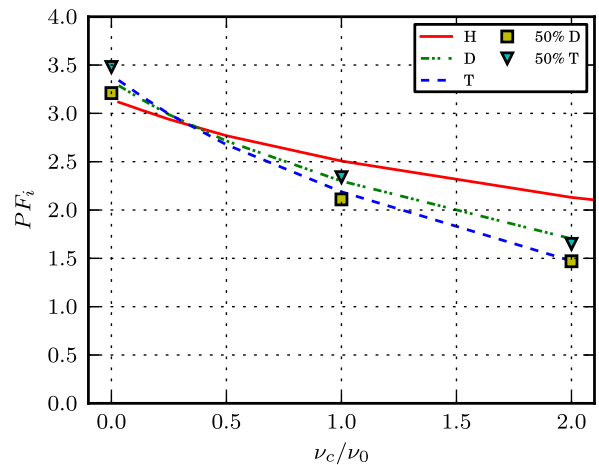

(a): scaling with collisionality

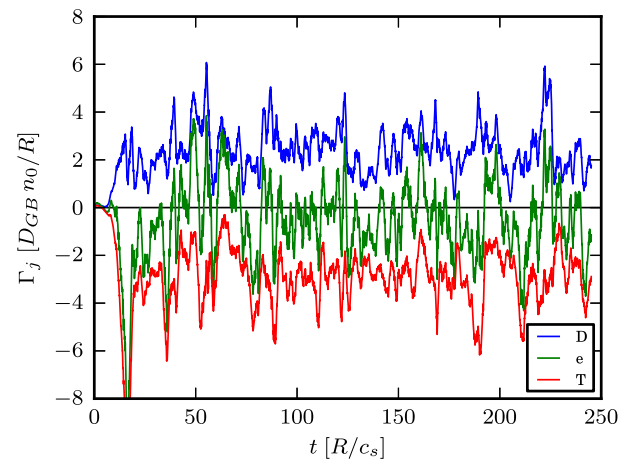

(a): time series

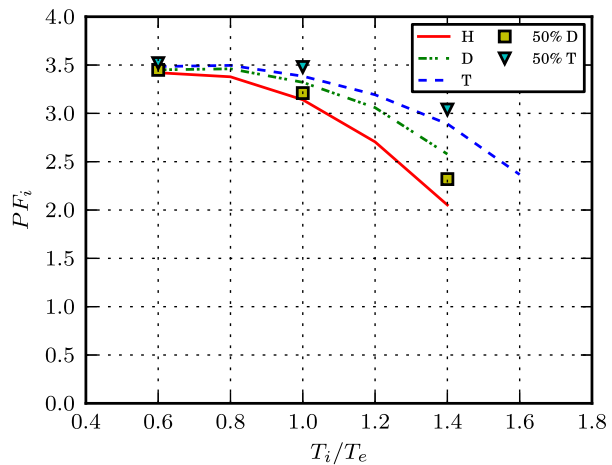

(b): scaling with temperature ratio

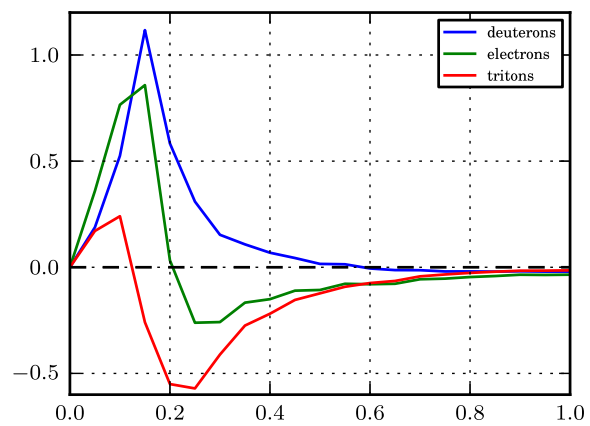

(b): flux spectra
FIG. 8. Scaling of main ion peaking with different parameters for the $\mathrm{CBC}$ (Table I), for $\mathrm{H}, \mathrm{D}, \mathrm{T}$, and a 50/50 mixture of $\mathrm{D}$ and $\mathrm{T}$ as main ions with $k_{\theta} \rho_{\mathrm{s}}=0.3$ in species units.
FIG. 9. Particles fluxes of species D, $\mathrm{T}$, and $e$ for CBC (Table I) with a $50 /$ 50 mixture of $\mathrm{D}$ and $\mathrm{T}$ as main ions. Evaluated at the zero flux gradient for the pure D case $\left(R / L_{n_{c}}=2.77\right)$ using NL GENE. normal $\mathrm{CBC}$ were compared with simulations where $\mathrm{D}$ was substituted for $\mathrm{H}$ and $\mathrm{T}$.

First, we review the known isotope effects on linear eigenvalues. Figure 7 displays the ITG eigenvalues in the collisionless case for $\mathrm{H}, \mathrm{D}$, and $\mathrm{T}$ in species units. The slight difference in eigenvalues obtained is due to the non-adiabatic electron response into which the mass ratio $\sqrt{m_{i} / m_{e}}$ enters, as discussed in Ref. 39.

The QL GENE background peaking versus collisionality is displayed in Fig. 8(a) for $k_{\theta} \rho_{\mathrm{s} i}=0.3$ in species units, corresponding to the peaks in the growthrate spectra. For $\nu_{e i}=0$, a slight difference in $P F$ is observed, with $P F_{\mathrm{T}}>P F_{\mathrm{D}}>P F_{\mathrm{H}}$. This is consistent with the asymmetry in $\mathrm{D}$ and $\mathrm{T}$ transport reported in Refs. 4 and 40. For larger values of the collisionality, however, the order is reversed.

Next, the effect of the ion mass on the stationary profile scaling with ion to electron temperature ratio $\left(T_{i} / T_{e}\right)$ is studied. In Fig. 8(b), the peaking factor is seen to decrease with increasing ion temperature, but in this case the lighter isotopes are more sensitive, showing a stronger decrease with $T_{i} / T_{e}$. The other parameter scalings discussed in Sec. IV show only a very weak isotope effect.

The scenario with a 50/50 mixture of $\mathrm{D}$ and $\mathrm{T}$ was also studied, and the simultaneous peaking of $\mathrm{D}$ and $\mathrm{T}$ calculated. The results were seen to follow the pure $\mathrm{D}$ and pure $\mathrm{T}$ results closely, albeit with the $\mathrm{T}$ profile approximately $10 \%$ more peaked than the $\mathrm{D}$ profile for all values of the collisionality; see Fig. 8(a). For the scan with $T_{i} / T_{e}$, the self-consistent case gave a larger difference in $\mathrm{D}$ and $\mathrm{T}$ peaking than the corresponding pure cases, as seen in Fig. 8(b). These results were corroborated by NL GENE simulations using the standard
CBC parameters, with the background electron density gradient corresponding to zero flux for the pure D case $\left(R / L_{n_{e}}=2.77\right)$. The results are shown in Fig. 9. For these parameters, the electron particle flux remained close to zero, while the deuterium flux was positive and the tritium flux negative, indicating a more peaked steady state $\mathrm{T}$ profile, and a less peaked D profile in the mixed scenario. The flow separation between $\mathrm{D}$ and $\mathrm{T}$ is also reproduced by the fluid model, as illustrated in Fig. 10, where the zero flux for electrons is balanced by a positive D flux and a negative T flux.

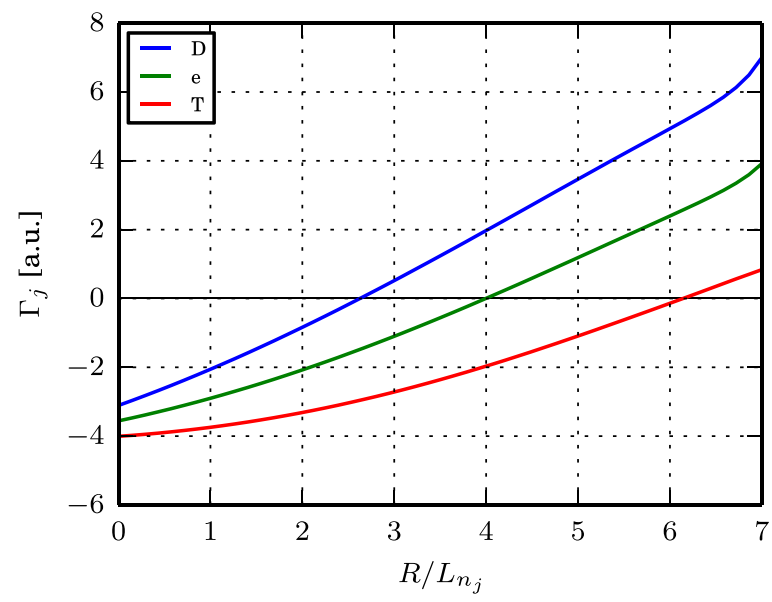

FIG. 10. Particles fluxes of species D, T, and $e$ for CBC (Table I) with a $50 / 50$ mixture of $\mathrm{D}$ and $\mathrm{T}$ as main ions, evaluated using the fluid model with $k_{\theta} \rho_{s}=0.3$. 


\section{CONCLUSIONS}

In the present paper, electron and impurity particles transport due to ITG/TE mode turbulence was studied using gyrokinetic simulations and a computationally efficient fluid model. The gyrokinetic simulations were performed with the code GENE and both QL and NL GENE simulations were compared and contrasted with the results of the fluid model. The focus was on a selfconsistent treatment of particle transport, where the stationary local profiles of electrons and impurities are calculated simultaneously corresponding to zero particle flux. The zero flux condition is relevant to the core region of tokamaks where the particle sources are absent or small. In addition, the density peaking in plasmas with varying isotope composition, from pure $\mathrm{H}, \mathrm{D}$, and $\mathrm{T}$ to 50/50 DT plasmas, was studied. Neoclassical contributions to the impurity transport, which may be relevant for high- $Z$ impurities, were neglected. The impurities, with impurity charge in the region $3 \leq Z \leq 42$, were included in low concentrations as trace species. The parameters were taken from the Cyclone base case, relevant for the core region of tokamaks, and scalings of the stationary profiles with magnetic shear, safety factor, electron-to-ion temperature ratio, collisionality, sheared toroidal rotation, plasma $\beta$, elongation, and triangularity were investigated.

It was shown that the stationary background density profile was sensitive in scans over magnetic shear, collisionality, elongation, temperature ratio, and plasma $\beta$. The selfconsistent treatment mainly enhanced the trends found in earlier works on impurity profile peaking assuming a fixed background. For collisionality, however, the increased peaking found for low collisionality was not accompanied by a corresponding increase in impurity peaking, making reactor relevant low collisionality plasma conditions favourable in this respect. A similar trend was seen see for $\beta$, where the background peaking was seen to be maximised for low $\beta$, while the higher charged impurities were only weakly affected or showed a lower peaking for lower $\beta$.

For safety factor, sheared toroidal rotation and triangularity on the other hand, the effects on the electron profile were weak and hence a selfconsistent treatment did not add significant new results to the previous investigations assuming a fixed background. For all considered cases, both the electron profile and the impurity profile were found to be inwardly peaked, with stationary density gradient scale lengths typically in the range $R / L_{n}=1.0-4.0$, i.e., substantially below neoclassical expectations. Furthermore, the electrons were consistently more peaked than the impurities.

In addition, a slight asymmetry between hydrogen, deuterium, and tritium with respect to profile peaking was obtained. The effect was more pronounced for high collisionality plasmas and large ion to electron temperature ratios. The effect of main ion mass on the stationary profiles discussed here is weak, but may result in a D-T fuel separation in a fusion plasma.

The fluid model was seen to reproduce the gyrokinetic results both qualitatively and quantitatively, though it was seen that the reliance one a single wave number for calculating the turbulence was a limitation. The same limitation applies to the QL GENE model used here, and a more complete QL treatment may give a better agreement. ${ }^{8,17}$ An extention of the fluid model to include a spectrum of wave numbers, as well as effects of shaping and rotation, is currently under development.

\section{ACKNOWLEDGMENTS}

The simulations were performed on resources provided by the Swedish National Infrastructure for Computing (SNIC) at PDC Centre for High Performance Computing (PDC-HPC), on the HELIOS supercomputer system at Computational Simulation Centre of International Fusion Energy Research Centre (IFERC-CSC), Aomori, Japan, under the Broader Approach collaboration between Euratom and Japan, implemented by Fusion for Energy and JAEA, and on the supercomputer JUROPA at Jülich Supercomputing Centre (JSC). This work was funded by a grant from The Swedish Research Council (C0338001) and by the European Union's Horizon 2020 research and innovation programme under grant agreement number 633053.

The authors would like to thank F. Jenko, T. Görler, M. J. Püschel, D. Told, and the rest of the GENE team at IPP-Garching for their valuable support and input.

${ }^{1}$ M. Fröjdh, M. Liljeström, and H. Nordman, Nucl. Fusion 32, 419 (1992)

${ }^{2}$ R. Basu, T. Jessen, V. Naulin, and J. J. Rasmussen, Phys. Plasmas 10, 2696 (2003).

${ }^{3}$ C. Angioni, A. G. Peeters, F. Jenko, and T. Dannert, Phys. Plasmas 12, 112310 (2005).

${ }^{4}$ C. Estrada-Mila, J. Candy, and R. W. Waltz, Phys. Plasmas 12, 022305 (2005).

${ }^{5}$ V. Naulin, Phys. Rev. E 71, 015402 (2005).

${ }^{6}$ C. Angioni and A. G. Peeters, Phys. Rev. Lett. 96, 095003 (2006).

${ }^{7}$ R. Guirlet, C. Giroud, T. Parisot, M. E. Puiatti, C. Bourdelle, L. Carraro, N. Dubuit, X. Garbet, and P. R. Thomas, Plasma Phys. Controlled Fusion 48, B63 (2006)

${ }^{8}$ C. Bourdelle, X. Garbet, F. Imbeaux, A. Casati, N. Dubuit, R. Guirlet, and T. Parisot, Phys. Plasmas 14, 112501 (2007).

${ }^{9}$ N. Dubuit, X. Garbet, T. Parisot, R. Guirlet, and C. Bourdelle, Phys. Plasmas 14, 042301 (2007).

${ }^{10}$ H. Nordman, T. Fülöp, J. Candy, P. Strand, and J. Weiland, Phys. Plasmas 14, 052303 (2007).

${ }^{11}$ C. Angioni and A. G. Peeters, Phys. Plasmas 15, 052307 (2008).

${ }^{12}$ H. Nordman, R. Singh, T. Fülöp, L.-G. Eriksson, R. Dumont, J. Anderson, P. Kaw, P. Strand, M. Tokar, and J. Weiland, Phys. Plasmas 15, 042316 (2008).

${ }^{13}$ C. Angioni, A. G. Peeters, G. V. Pereverzev, A. Bottino, J. Candy, R. Dux, E. Fable, T. Hein, and R. E. Waltz, Nucl. Fusion 49, 055013 (2009).

${ }^{14}$ C. Angioni, E. Fable, M. Greenwald, M. Maslov, A. G. Peeters, H. Takenaga, and H. Weisen, Plasma Phys. Controlled Fusion 51, 124017 (2009).

${ }^{15}$ Y. Camenen, A. G. Peeters, C. Angioni, F. J. Casson, W. A. Hornsby, A. P. Snodin, and D. Strintzi, Phys. Plasmas 16, 012503 (2009).

${ }^{16}$ S. Moradi, M. Z. Tokar, R. Singh, and B. Weyssow, Nucl. Fusion 49, 085007 (2009)

${ }^{17}$ E. Fable, C. Angioni, and O. Sauter, Plasma Phys. Controlled Fusion 52, 015007 (2010).

${ }^{18}$ T. Fülöp, S. Braun, and I. Pusztai, Phys. Plasmas 17, 062501 (2010).

${ }^{19}$ S. Futatani, X. Garbet, S. Benkadda, and N. Dubuit, Phys. Rev. Lett. 104, 015003 (2010).

${ }^{20}$ T. Hein and C. Angioni, Phys. Plasmas 17, 012307 (2010).

${ }^{21}$ C. Angioni, R. McDermott, E. Fable, R. Fischer, T. Pütterich, F. Ryter, G. Tardini, and the ASDEX Upgrade Team, Nucl. Fusion 51, 023006 (2011).

${ }^{22}$ H. Nordman, A. Skyman, P. Strand, C. Giroud, F. Jenko, F. Merz, V. Naulin, T. Tala, and the JET-EFDA contributors, Plasma Phys. Controlled Fusion 53, 105005 (2011).

${ }^{23}$ A. Skyman, H. Nordman, and P. Strand, Phys. Plasmas 19, 032313 (2012).

${ }^{24}$ A. Skyman, H. Nordman, and P. Strand, Nucl. Fusion 52, 114015 (2012). 
${ }^{25}$ F. J. Casson, R. M. McDermott, C. Angioni, Y. Camenen, R. Dux, E. Fable, R. Fischer, B. Geiger, P. Manas, L. Menchero, G. Tardini, and the ASDEX Upgrade Team, Nucl. Fusion 53, 063026 (2013).

${ }^{26}$ A. Skyman, L. Fazendeiro, D. Tegnered, H. Nordman, J. Anderson, and P. Strand, Nucl. Fusion 54, 013009 (2014).

${ }^{27}$ See http://www.ipp.mpg.de/ fsj/gene/ for information on the GENE code.

${ }^{28}$ F. Jenko, W. Dorland, M. Kotschenreuther, and B. N. Rogers, Phys. Plasmas 7, 1904 (2000).

${ }^{29}$ F. Merz, "Gyrokinetic simulation of multimode plasma turbulence," Ph.D. dissertation (Westfälischen Wilhelms-Universität Münster, 2008).

${ }^{30} \mathrm{~J}$. Weiland, Collective Modes in Inhomogeneous Plasmas (IoP Publishing, Bristol, UK, 2000).

${ }^{31}$ G. Cennachi and A. Taroni, "JETTO: A free-boundary plasma transport code," Technical Report JET-IR(88)03 (JET Reports, 1988).

${ }^{32}$ A. M. Dimits, G. Bateman, M. A. Beer, B. I. Cohen, W. Dorland, G. W. Hammett, C. Kim, J. E. Kinsey, M. Kotschenreuther, A. H. Kritz, L. L.
Lao, J. Mandrekas, W. M. Nevins, S. E. Parker, A. J. Redd, D. E. Shumaker, R. Sydora, and J. Weiland, Phys. Plasmas 7, 969 (2000).

${ }^{33}$ A. Hirose, L. Zhang, and E. Elia, Phys. Rev. Lett. 72, 3993 (1994)

${ }^{34}$ C. Angioni, Y. Camenen, F. J. Casson, E. Fable, R. M. McDermott, A. G. Peeters, and J. E. Rice, Nucl. Fusion 52, 114003 (2012).

${ }^{35}$ F. J. Casson, A. G. Peeters, C. Angioni, Y. Camenen, W. A. Hornsby, A. P. Snodin, and G. Szepesi, Phys. Plasmas 17, 102305 (2010).

${ }^{36}$ R. L. Miller, M. S. Chu, J. M. Greene, Y. R. Lin-Liu, and R. E. Waltz, Phys. Plasmas 5, 973 (1998).

${ }^{37}$ C. Angioni, J. Candy, E. Fable, M. Maslov, A. G. Peeters, R. E. Waltz, and H. Weisen, Phys. Plasmas 16, 060702 (2009).

${ }^{38}$ T. Hein, C. Angioni, E. Fable, and J. Candy, Phys. Plasmas 17, 102309 (2010).

${ }^{39}$ I. Pusztai, J. Candy, and P. Gohil, Phys. Plasmas 18, 122501 (2011).

${ }^{40}$ H. Nordman, P. Strand, A. Eriksson, and J. Weiland, Plasma Phys. Controlled Fusion 47, L11 (2005). 